\title{
Una registrazione neo-sumerica di orzo da Nippur
}

\author{
Emanuela DI PASQUALE \\ Università di Messina
}

Il testo del quale si presenta qui l'edizione è un registro di assegnazioni di orzo. La tavoletta proviene dal mercato antiquario e appartiene alla collezione privata del Dr. Massimo Baldacci di Roma. Chi scrive ringrazia il proprietario della tavoletta per averne generosamente consentito la pubblicazione e la Drssa. Maria Elena Milone per averne preparato l'autografia.

Colore: grigio.

Dimensioni: $70 \times 45 \times 23 \mathrm{~mm}$.

Data: ŠS5/VI/18.
r. 1) 10.4.4 še gur
10 gur e 280 sila di orzo:
2) še Lú- ${ }^{\mathrm{d} E n-l i ́ l-l a ́ ~}$
orzo di Lu-Enlila.
3) linea anepigrafa
4). šà-bi-ta 3.4 .0 gur
Di questi, 3 gur e 240 sila,
5) á lú-hun-gá
come pagamento di lavoratori ingaggiati,
6) Ur- ${ }^{d} \mathrm{Ba}-u$ per Ur-Bau;
7) 3.1.0 gur $\operatorname{Ir}_{11}$-dam / dumu Ur- ${ }^{\mathrm{d}}$ Nin-urta
3 gur e 60 sila per Irdam, figlio di Ur-
Ninurta;
8) [...] gur A-ba-at-ì-תí
[...] gur per Abāt-ilī;
9) [... g]ur Lugal-nam-<tar>-re [...] gur per Lugal-namtarre;

Sefarad 63 (2003) págs. 141-145

(c) CSIC

ISSN 037-0894 
v. 1) $\left[\ldots{ }^{\mathrm{d}}\right]$ Nin-šubur-an-/dùl

2) $[\ldots] A ́$ - bí-lí

3) $0.1 .0 \mathrm{Nu}$-úr-ì-lí / šà-gu

4) 0.0 .3 Ur-Du ${ }_{6}-\mathrm{kù} \mathrm{sipa}$

5) 0.1.3 ̌̌ $u$ - $A d a d$ šeš $E n$ $m i-E \check{s}_{4}$-tár

6) $0.1 .0 \mathrm{~A}-\mathrm{si}-\mathrm{ma}_{2}-[\mathrm{a}]$

7) šu-nigín 11.2.4 še gur

8) ba-zi 0.2.4 (sic $\left.{ }^{\prime}\right) \quad$ íb-tag

9) ki Ad-da-kal-la

10) iti kin- ${ }^{d}$ Inanna $u_{4}-18$ / bazal

11) mu-ús-sa bàd-Mar-/tu ${ }^{\mathrm{ki}}$ badù

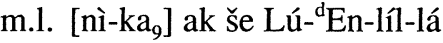

[...] per Ninšubur-andul;

[...] per Abili;

60 sila per Nūr-ilī, il bovaro;

30 sila per Ur-Duku, il pastore;

90 sila per Šū-Adad, il fratello di Enmi-

Eštar;

60 sila per Asima'a.

Totale: 11 gur e 160 sila di orzo prelevati; 180 sila sono stati trascurati.

'Ufficio' di Addakalla.

Mese della Festa-kin di Inanna, (di cui) 18 giorni sono trascorsi.

Anno dopo quello in cui la muraglia dei

Martu fu eretta.

Resoconto dell'orzo di Lu-Enlila.

Il testo può essere diviso nelle seguenti sezioni:

r.1-2: è registrato un quantitativo di orzo con il relativo amministratore, Lu-Enlila;

r.4-v.6: sono elencati i dieci destinatari di questo orzo;

v.7-9: è calcolato il totale delle quantità prelevate (ba-zi) e la differenza tra questo e la somma a disposizione all'inizio del prelievo. Qui possiamo leggere 2 bariga ovvero 2 bariga e, parzialmente cancellati, 4 ban, ma non gli attesi 3 bariga.

v.9 - marg. lat.: dopo la data, la formula sul margine della tavoletta, «resoconto dell'orzo di Lu-Enlila», indica il tipo di registrazione fatta nel testo. 
Per quanto riguarda la provenienza della tavoletta, è verosimile una sua appartenenza a un archivio di Nippur ${ }^{1}$, suggerita dal nome del mese e da alcuni antroponimi (Ur-Ninurta, Ur-Duku).

Quanto ai personaggi che compaiono nel testo, il Lu-Enlila, responsabile dell'orzo potrebbe essere identificato con il personaggio che compare in molti contratti di prestito, sia d'orzo, sia d'argento da Nippur di questo periodo, a volte associato ad altri nomi che pure compaiono nel nostro testo. Per quanto riguarda, in particolare, Addakalla, il personaggio dal quale è preso in consegna l'orzo del nostro testo, abbiamo:

TMH NF I/2 98 (ŠS9/xi/11), in cui Lu-Enlila è testimone in un contratto di prestito di orzo da parte di Addakalla;

NRVN 168 (IS1/xii/-), in cui Lu-Enlila è testimone in un contratto di prestito di orzo ricevuto da Addakalla;

TMH NF I/2 101 (ŠS9/X/-), in cui Lu-Enlila presta orzo ad Addakalla.

1 Per una sintetica presentazione delle caratteristiche dei testi economicoamministrativi di Nippur, in contrapposizione a quelli provenienti da altre province dell'impero di Ur III, cf. da ultimo W. SALlABERGER e A. WESTENHOLZ, Mesopotamien. Akkade-Zeit und Ur III-Zeit, OBO 160/3 (Freiburg 1999) pp. 330-331. 

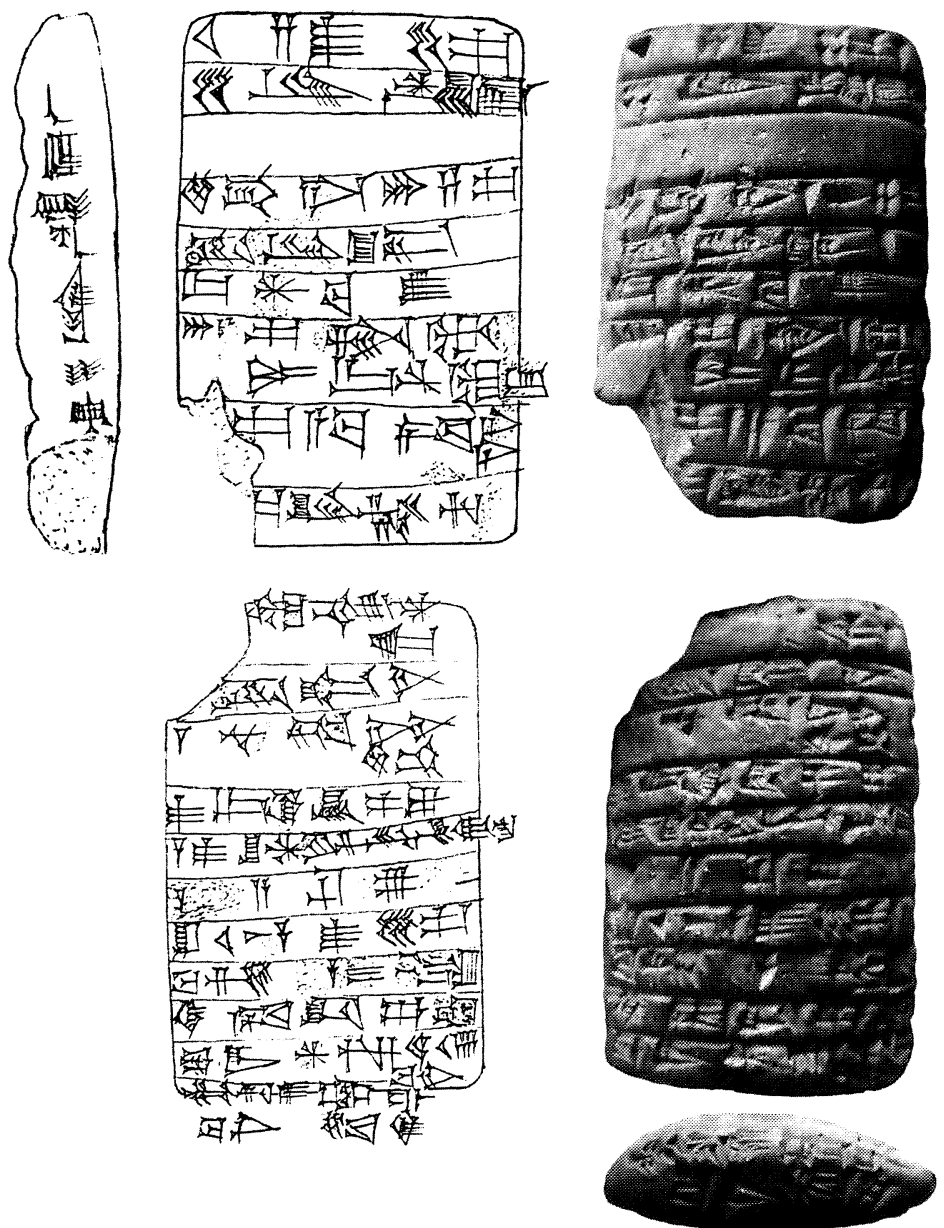


\section{RESUMEN}

El artículo presenta la edición de un texto cuneiforme de época neo-sumeria procedente de la ciudad de Nippur. La tablilla registra un balance contable de asignaciones de cebada a diversos individuos, bajo la responsabilidad de Lu-Enlila. El dcoumento está datado en el sexto mes del año 5 de reinado de Shu-Suen.

PALABRAS CLAVE: Texto sumerio, Nippur, administración, balance contable.

\section{SUMMARY}

This article presents the edition of a Neo-Sumerian cuneiform text from Nippur. The tablet records a balanced account of barley distributed to several people under the supervision of Lu-Enlila, and is dated to the sixth month of Shu-Suen's fifth year.

KEYWORDS: Sumerian text, Nippur, administration, balanced account. 